\title{
Bilingualism and Speech Understanding in Noise: Auditory and Linguistic Factors
}

DOI: $10.3766 /$ jaaa.17082

\author{
Erika Skoe*t+ \\ Kateryna Karayanidi*+
}

\begin{abstract}
Background: Bilingual speakers often have difficulty understanding speech in noisy and acoustically degraded conditions.

Purpose: The first aim was to examine the potential source(s) of the difficulties that English-proficient bilingual listeners experience when hearing English speech in noise. The second aim was to assess how bilingual listeners perform on a battery of central auditory processing tests.
\end{abstract}

Research Design: A mixed design was used in this study.

Study Sample: Normal-hearing college students $(n=24)$ participated in this study. The bilingual participants $(n=12)$ self-reported that they learned a second language before age 9 and the monolingual participants reported that they only knew American English. All participants considered themselves to be native speakers of American English.

Data Collection and Analysis: Participants were administered the Revised Speech Perception in Noise (R-SPIN) test to assess whether bilingual listeners' speech understanding in noise reflects auditory factors, linguistic factors, or a combination of the two. To minimize the influence of short-term memory and motor movements, only the final word of a sentence is repeated for this test. Sentence-final words were presented in two linguistic contexts: in the high-predictability condition, the final word can be deduced from the context created by the preceding words, and in the low-predictability condition, it cannot. The R-SPIN test was administered at two signal-to-noise ratios (SNRs) ( 0 and $3 \mathrm{~dB}$ ). In addition, the participants were given a reading comprehension test to measure their ability to use context when linguistic stimuli are delivered to the visual, not auditory, modality. The central auditory test battery consisted of three tests: Competing Sentences, Dichotic Digits, and NU-6 Time-Compressed Speech with Reverberation. All test materials were given in American English.

Results: The bilingual and monolingual groups performed similarly in the low-context condition of the R-SPIN test. However, in comparison to the age-matched monolingual group, the bilingual group did not derive the same level of benefit from contextual cues, as seen by a smaller improvement in performance between the low- and high-predictability R-SPIN conditions. The bilingual and monolingual groups showed a similar decrement in performance when the SNR dropped. In addition, bilingual individuals underperformed on the Competing Sentences test when instructed to attend to the left ear. However, the bilingual and monolingual groups performed equally well on the reading comprehension test, as well as on the Time-Compressed Speech with Reverberation and Dichotic Digits tests.

Conclusions: We show that individuals who are exposed to two languages from an early age, and selfreport as having a high level of proficiency in English, perform like their monolingual counterparts in acoustically degraded conditions where context is not facilitative, but they underperform in conditions where sentence-level linguistic context is facilitative to understanding. We conclude that deficits observed in noise are likely not due to a perceptual deficit or a lack of linguistic competence, but instead reflect a linguistic system that performs inefficiently in noise. In addition, we do not find evidence of an auditory processing weakness or advantage in our bilingual cohort; however, the use of speech materials to assess auditory processing is a confound.

*Department of Speech, Language, and Hearing Sciences, University of Connecticut, Storrs, CT; †Department of Psychological Sciences, Cognitive Sciences Program, Connecticut Institute for Brain and Cognitive Sciences, University of Connecticut, Storrs, CT; $¥$ University of Connecticut, Storrs, CT

Corresponding author: Erika Skoe, University of Connecticut, Storrs, CT 06129; Email: erika.skoe@uconn.edu 
Key Words: bilingual, speech in noise

\begin{abstract}
Abbreviations: ANOVA = analysis of variance; CAP = central auditory processing; CAPD = central auditory processing disorder; CST = Competing Sentences Test; DDT = Dichotic Digits Test; NEL = non-English language; R-SPIN = Revised Speech Perception in Noise; SD = standard deviation; SES = socioeconomic status; SIN = speech in noise; SNR = signal-to-noise ratio; SRT = speech recognition threshold; SUN = speech understanding in noise
\end{abstract}

\section{INTRODUCTION}

$\mathrm{T}$ here is a preponderance of evidence that bilingual speakers have more difficulty understanding speech in noisy and acoustically degraded conditions than their monolingual counterparts (Mayo et al, 1997; Rogers et al, 2006; Bradlow and Alexander, 2007; Shi, 2010; 2012; Tabri et al, 2011; Hervais-Adelman et al, 2014; Krizman et al, 2016). This increased perceptual difficulty is akin to having a mild hearing loss, even for listeners with clinically normal audiometric thresholds. Lucks Mendel and Widner (2016) suggest that the bilingual disadvantage for speech understanding in noise (SUN) is the consequence of "auditory processing degradation," although other work suggests a bilingual advantage for low-level auditory processing (Krizman et al, 2016). Bilingualism, thus, provides an interesting test case for examining the relative roles of auditory versus linguistic contributions to SUN. The present study explores the potential source(s) of the perceptual difficulties that bilingual individuals experience when listening to speech in noise (SIN), and we specifically focus on bilingual listeners who consider themselves to be proficient in the test language.

SIN testing is a routine part of audiological practice. SIN tests are an attractive clinical tool because they access the most common complaint that brings a patient to the audiologist in the first place, namely, difficulty understanding speech in noisy backgrounds. The tests in most wide-scale use include the Hearing in Noise Test (Nilsson et al, 1994), the Words in Noise Test (Wilson et al, 2003), and the QuickSIN test (Killion et al, 2004) (For a review see, Lagacé et al, 2010). Most of these tests can be administered in a matter of minutes and require the listener to repeat a single word or the entire sentence. The requirement to have the listener repeat what they heard as a way of indexing perceptual acuity is a complication to test interpretation because performance depends not only on the listener's auditory percept, but also on working memory, the ability to form and execute speech motor plans, top-down linguistic knowledge, among other variables. Most of these SIN tests also lack the specificity to isolate whether the listener's weakness is due to auditory factors, linguistic factors, cognitive, memory factors, motor factors (etc.), or some combination thereof. Understanding which factor or set of factors contribute to decreased performance in noise is essential for providing appropriate clinical counseling as well as compensatory and/or remediation strategies.

Lagacé et al (2010) proposed using the Revised Speech Perception in Noise (R-SPIN) test (Bilger et al, 1984) to evaluate whether decreased speech perception in noise is the result of weak auditory processing, weak language processing, or a combination of the two. It has been argued that both types of impairments can manifest in poor SUN, although the underlying mechanisms are presumed to be different. To minimize the influence of short-term memory and motor movements, only the final word of a sentence is repeated in the R-SPIN test. In addition, the target words are presented as part of a design that uses two levels of linguistic predictability. In the high-predictability condition, the final word can be deduced from the context created by the preceding words in the sentence, and in the low-predictability condition, it cannot. For example, "The lion gave an angry roar" (high-predictability final word) versus "He is thinking about the roar" (low-predictability final word). The high- and low-predictability conditions can then each be presented with different levels of masking to manipulate the signal-to-noise ratio (SNR), creating low-SNR and high-SNR conditions. Comparing performance on the low- and high-SNR conditions can give insight into the auditory processes contributing to SUN. According to Lagacé et al (2010), this factorial design, which manipulates both predictability and SNR, provides a method for dissociating auditory processing from linguistic processing. The authors posit that a listener with a "pure" auditory processing issue (i.e., an auditory processing problem without concomitant language problem) will perform more poorly than a typical listener as the SNR becomes less favorable; however, they will receive the same, if not potentially greater, benefit when the word can be deduced from the linguistic context. Partial evidence for this pattern of findings can be found in Lagacé and colleague's small-scale study of children with central auditory processing disorder (CAPD) (Lagacé et al, 2011). Lagacé et al (2011), argue that if reduced SUN is the result of languagespecific processes, the benefit from context will be small (if not at all) compared with typical listeners, but the listener will not be inordinately affected by changes in SNR. If an individual underperforms as the SNR decreases and they also do not benefit from context in the typical manner, this, Lagacé et al (2010) argue, should be taken as evidence that both auditory and 
linguistic processes are contributing to poor perception of SIN. As a logical extension of the argument made by Lagacé et al (2010), if a listener experiences less of a performance decrement than typical listeners when the SNR is decreased, this would be an indication of an auditory processing advantage.

We focused on bilingual adults who self-reported as being highly proficient speakers of English. The bilingual college students in our sample learned English in combination with another language from a young age (before age 9), they were proficient at the native level in both their languages (self-report), were college students at an American university, and spoke English without a noticeable accent. Thus, the bilingual participants in our sample are likely to have been mistaken for monolingual English speakers during routine, daily communication by other native, monolingual speakers of English. For listeners who are still learning the test language, or who consider themselves to be nonnative speakers of the test language, poor performance on SIN tests can be explained by an underdeveloped knowledge of the target language that results from reduced exposure to that language compared with monolingual speakers of that language. For some bilingual listeners, this reduced language exposure is the necessary consequence of having learned the target language at a later age (Shi, 2010) and in most examples cited in the literature, underperformance on SIN tasks can be attributed to measurable differences in language proficiency, years of exposure, or the degree of balance between the two languages (Shi, 2010; 2012). The present study asks whether such disadvantages persist even when the listener has achieved a high level of language proficiency in the target language. Schmidtke (2016) recently reported that bilingual speakers have poorer word recognition ability on a modified version of the R-SPIN, even after controlling for differences in verbal ability between the bilingual and monolingual subsamples. This lead to the hypothesis that underperformance on SIN tasks is an inevitable consequence of splitting resources across multiple languages (Schmidtke, 2016).

The primary aim of the present study was to explore the auditory and/or linguistic basis of the bilingual disadvantage for SUN using the R-SPIN test. The second aim of our study was to assess the auditory processing skills of our bilingual listeners using a battery of tests that are commonly used in a clinical setting to diagnose CAPD. The CAPD test battery included TimeCompressed Speech with Reverberation, Dichotic Digits, and Competing Sentences. These tests, like the R-SPIN, use linguistic stimulation, but unlike the R-SPIN, they do not use background babble as a distractor. Krizman et al (2016) recently hypothesized that bilingual listeners develop stronger auditory processing skills as a way to compensate for the challenges they face for SUN. This hypothesis emerged from a recent string of studies showing superior performance to monolingual listeners for processing auditory signals, including signals that are masked by noise. Krizman et al (2016), reported that adolescents who learned two language from an early age had lower thresholds for simultaneous and backward masking tasks compared with monolingual counterparts. Montagni and Peru (2011) provide additional evidence that early exposure to a second language confers an advantage to auditory processing tasks across both linguistic and musical stimuli. Moreover, early exposure to two languages (for both children and adults) has also been associated with more robust (pre-attentive) neural processing of speech sounds in both quiet and background babble conditions (Krizman et al, 2012; 2014; 2015; Skoe et al, 2017).

In addition to this recent evidence of an auditory processing advantage in bilingual individuals, there is compelling evidence that bilingual individuals have stronger executive functions, in particular, stronger inhibition of task-irrelevant stimuli that are both auditory and visual in nature (Soveri et al, 2011; Krizman et al, 2012; Bak et al, 2014; Bialystok, 2015). For bilinguals, inhibitory control has been theorized to emerge as a by-product of needing to suppress one language when the other is the target language (Green, 1998), although a more modern account is that increased inhibitory control is the result of needing to monitor which language to produce in different communication settings (Costa et al, 2009). There is a small body of literature suggesting that increased inhibitory control contributes to heightened dichotic processing in bilingual listeners (Soveri et al, 2011; Gresele et al, 2013). This literature predicts that bilingual listeners should outperform monolingual listeners on tests of dichotic listening, such as Competing Sentences and Dichotic Digits. When listening to dichotic speech stimulation, there is a bias toward listening to the right ear, even when instructed to attend to the left ear. This rightear bias, which is well described in the scientific literature, is presumed to be the outcome of the right ear having a more direct pathway to speech-specialized regions in the left temporal lobe than the left ear. As a consequence of this circuitry, attending to the left ear is theorized to require more executive processing than attending to the right ear under dichotic stimulation (reviewed in Hugdahl et al, 2009). By this argument, heightened executive function is expected to boost bilingual listeners' ability to attend to the left ear. However, the whole notion that bilinguals have advantages in executive function has recently been called into question (Paap et al, 2014; 2015). In addition, because central auditory processing (CAP) tests are intended to assess impaired (not extraordinary) auditory processing and they typically use speech materials, they may lack 
the sensitivity to observe bilingual advantages for auditory processes and/or executive function.

\section{METHODS}

\section{Participants}

The study included 12 monolingual speakers (9 females) and 12 bilingual speakers (9 females), all students at the University of Connecticut with a negative history of hearing impairment and negative history of speech or language pathology. All procedures were approved by the University of Connecticut Institutional Review Board. Participants gave their written informed consent to participate, and they were either paid or compensated through course extra credit for their participation (their choice). Consent and all testing materials and instructions were delivered in American English. Testing was conducted in a 1.5- to 2-hour session, with breaks given between tests. All testing was administered by the second author, an EnglishRussian bilingual, who learned English at age 11. The potential confounds of having a bilingual test administrator are addressed in the Clinical Implications section of the "Discussion."

Participants completed a survey of their bilingual background and language exposure. The survey was a modified version of the survey developed by GarcíaSierra et al (2012). In the survey, participants rated their ability to use English and all other languages that they knew, using a Likert scale from 1 to 10, with 10 being labeled as "expert." To validate the consistency of their ratings, at a later point in the survey, the participants were asked to indicate whether they considered themselves to have native-like proficiency for English and each non-English language (NEL). With respect to English, all participants rated themselves as 9 or 10 and described their proficiency as "nativelike." The survey also included questions about confi- dence reading in their NEL. In addition, participants were given a musical training questionnaire adapted from the one created by Kraus and colleagues (Slater and Kraus, 2016) because of the literature showing an association between musical training and SUN advantages (Parbery-Clark et al, 2009; Bak et al, 2014; Slater et al, 2015).

On the bilingual background survey, participants were instructed to indicate the degree to which they were exposed to English versus their NEL at different points in their life, broken down in increments of three years (i.e., 0-3, 3-6, 6-9, etc.), using a rating of $0 \%, 25 \%$, $50 \%, 75 \%$, or $100 \%$, with $100 \%$ indicating exposure to NEL only and 0\% indicating exposure to English only. The monolinguals indicated that they were not exposed to a language other than English during their day-today communication at any point in their life. For all but four bilingual participants, exposure to English and the NEL began during the first 3 years of life. For the remaining four, English was learned as the second language after age 3 but before age 9 . In all cases, the NEL was spoken by one or both of their parents. For two of the bilingual participants, one parent was a native speaker of English and the other was a native speaker of the NEL. At the time of testing, average exposure to the NEL was $29.1 \%$ (standard deviation $[\mathrm{SD}]=14.43 \%)$. In terms of language use, all participants were English dominant at the time of testing. The NELs included Bangla, Japanese, Mongolian, Polish, Portuguese $(\times 2)$, Serbian, Spanish $(\times 3)$, Tamil, and Telugu (Table 1).

The bilingual and monolingual groups were matched with respect to age, self-rated English proficiency, bilateral pure-tone averages $(0.5,1,2 \mathrm{kHz})$, as well as maternal education, a commonly used index of socioeconomic status (SES) ( $p>0.05$ for all comparisons) (Table 2 ). In addition, the groups were matched with respect to the number of years of musical training $(\sim 3.4$ years on average for the monolingual group and $\sim 4.5$ years on

Table 1. Bilingual Group Demographics

\begin{tabular}{|c|c|c|c|c|c|c|c|}
\hline $\begin{array}{l}\text { Age Window of First } \\
\text { Exposure to English (Years) }\end{array}$ & Age & Sex & NEL & $\begin{array}{c}\text { English } \\
\text { Proficiency }(/ 10)\end{array}$ & $\begin{array}{c}\text { NEL } \\
\text { Proficiency }(/ 10)\end{array}$ & $\begin{array}{l}\text { Literate } \\
\text { in NEL }\end{array}$ & $\begin{array}{c}\text { Live Outside } \\
\text { United States (Years) }\end{array}$ \\
\hline$\overline{0-3}$ & 26 & $\mathrm{~F}$ & Spanish & 10 & 8 & Yes & \\
\hline $0-3$ & 21 & $\mathrm{~F}$ & Portuguese & 10 & 9 & Yes & \\
\hline $0-3$ & 19 & $\mathrm{~F}$ & Telugu & 9 & 8 & No & \\
\hline $0-3$ & 22 & $\mathrm{~F}$ & Spanish & 10 & 10 & Yes & 2 \\
\hline $0-3$ & 20 & $\mathrm{~F}$ & Spanish & 10 & 7 & No & \\
\hline $0-3$ & 20 & $\mathrm{~F}$ & Mongolian & 10 & 9 & No & 2 \\
\hline $0-3$ & 23 & $\mathrm{~F}$ & Bangla & 10 & 8 & No & \\
\hline $0-3$ & 18 & M & Tamil & 10 & 8 & No & 3 \\
\hline $3-6$ & 22 & M & Japanese & 10 & 9 & Yes & 4 \\
\hline $3-6$ & 20 & $\mathrm{~F}$ & Polish & 10 & 7 & No & \\
\hline $3-6$ & 20 & $\mathrm{~F}$ & Serbian & 10 & 7 & Yes & 2 \\
\hline $6-9$ & 24 & M & Portuguese & 9 & 10 & Yes & 14 \\
\hline
\end{tabular}


Table 2. Group Means and SDs for Age, Self-Rated Current Proficiency of L1, L2, and English, Musical Training, Maternal Education, and Pure-Tone Audiometric Thresholds (Pure-Tone Averages [PTAs])

\begin{tabular}{llrrr}
\hline & & $\mathrm{N}$ & Mean & $\mathrm{SD}$ \\
\hline Age (years) & Mono & 12 & 20.30 & 1.22 \\
& $\mathrm{Bi}$ & 12 & 21.31 & 2.22 \\
L1 self-rated proficiency (/10) & Mono & 12 & 9.92 & 0.29 \\
& $\mathrm{Bi}$ & 12 & 9.25 & 1.22 \\
L2 self-perceived proficiency (/10) & Mono & 12 & 3.17 & 1.64 \\
& $\mathrm{Bi}$ & 12 & 8.92 & 1.00 \\
English self-rated proficiency (/10) & Mono & 12 & 9.92 & 0.29 \\
& $\mathrm{Bi}$ & 12 & 9.75 & 0.45 \\
Musical training (years) & Mono & 12 & 3.42 & 4.33 \\
& $\mathrm{Bi}$ & 12 & 4.50 & 3.82 \\
Maternal education (years) ${ }^{*}$ & Mono & 12 & 15.33 & 2.95 \\
& $\mathrm{Bi}$ & 11 & 14.73 & 3.26 \\
PTA: Right (dB HL) & Mono & 12 & 12.22 & 5.92 \\
PTA: Left (dB HL) & $\mathrm{Bi}$ & 12 & 10.56 & 3.65 \\
& Mono & 12 & 10.00 & 4.55 \\
& $\mathrm{Bi}$ & 12 & 8.75 & 3.42
\end{tabular}

${ }^{*}$ One of the bilingual participants did not answer this question as a result of a photocopying error.

average for the bilingual group) (Table 2). In the case of the bilingual participants, all but one reported having received voice or instrumental training in the past but none were presently active in music activities. For the monolingual group, two were still active in music activities and five reported never having received any musical training in the past.

\section{Test Battery}

All testing was completed in a double-walled sound booth (IAC Acoustics) in the Auditory Brain Research Laboratory at the University of Connecticut. Before any SIN testing, participants were first verified to have normal otoscopy, normal bilateral air conduction thresholds <25-dB HL for octaves $125-8000 \mathrm{~Hz}$ (GSI 61 audiometer), and normal outer hair cell function as confirmed by a distortion product otoacoustic emissions screening protocol performed using a handheld screener (Madsen Alpha OAE + Screener, GN Otometrics). Speech recognition thresholds (SRTs) were obtained using the modified Hughson-Westlake method for the right and left ears (separately) via monitored live voice, after first familiarizing the participants with the spondee words: ice cream, baseball, toothbrush, airplane, outside, mushroom, and sunshine. Binaural SRTs were obtained in the same manner, except spondees were presented to both ears at the same time via monitored live voice. The test order was right SRT, left SRT, and then binaural SRT. All subsequent test materials were delivered relative to the SRT; for binaural tests, such as the R-SPIN, binaural SRTs were used. The tests were administered in the following order:
Competing Sentences (right ear first), Dichotic Digits, NU-6 Time-Compressed Speech with Reverberation (right ear first), R-SPIN, and then the Passage Comprehension Test (Woodcock-Johnson Mastery Tests of Achievement IIII, WRMT-III). The Competing Sentences, Dichotic Digits, and Time-Compressed Speech tests are distributed by Auditech, Inc. (St. Louis, MO) and test administration followed recommended guidelines. Test materials were delivered from a desktop computer routed through a two-channel GSI 61 audiometer to ER2 insert earphones.

\section{Competing Sentences Test (CST) (Willeford, 1978)}

This test of binaural separation includes 20 pairs of simple sentences spoken by a man, with the sentences being six to seven words in length. For each pair, one sentence is presented to the right ear and the other is presented simultaneously to the left ear. The two sentences in the pair have a similar theme. For illustrative purposes, here are two example pairs: (a) "I was late today" and "This watch keeps good time" and (b) "We had to repair the car" and "We usually take a taxi." The participant was instructed to listen and repeat back the sentence presented to one ear while ignoring the sentence presented to the other. For the first ten sentences, the participant is instructed to repeat back the entire sentence presented to the right ear and for the final set of ten sentences, they were instructed to repeat back the entire sentence presented to the left ear. The target sentence was presented at 35-dB SL (re: SRT) and the competing sentence was presented at 50-dB SL (re: SRT). Each sentence is worth ten points (2.5 per word), and there are ten sentences, yielding a total possible maximum score of 100. For assessing CAPD, a score $<90 \%$ for the right ear and $<90 \%$ for the left ear is considered abnormal for adults (11+ years).

\section{Dichotic Digits Test (DDT) (Musiek, 1983)}

In this test of binaural integration, the participant repeats what he/she heard in both ears. For each of the 20 trials, two digits are presented to each ear (80 total digits, 40 per ear) at 50-dB SL (re: SRT). The digits include monosyllabic numbers between 1 and 10 (i.e., all numbers except 7). The participant is instructed to listen to both ears and repeat the numbers without concern about the order. Participants are encouraged to guess if they are not sure of what they heard. Before administering the test material, the participant is given three practice trials. To score the test, the number of correctly repeated digits is totaled for each ear separately ( 40 points per ear) and converted to a percentage. For assessing CAPD, a score $<90 \%$ for the right ear or $<90 \%$ for the left is considered abnormal for adults. 
Like Competing Sentences, a right-ear advantage is expected. Compared with the other tests that were administered, this has a relatively light linguistic load.

\section{NU-6 Time-Compressed Speech with Reverberation (Wilson et al, 1994)}

In this monaural test, the speech materials, spoken by a female, are time-compressed (45\%) with $0.3 \mathrm{sec}$ of reverberation. Participants are told that they will hear a woman's voice and that she will sound as if she is in a gymnasium. They are instructed to verbally repeat the word that they hear the woman say. Each sentence starts with the carrier phase "Say the word __ with the final word being drawn from the NU-6 list of words. In this test, the final word cannot be derived from the preceding word. For each sentence, the listener must repeat back the final word that they heard and guess if they are uncertain. Sentences were presented at 50-dB SL (re: SRT), with 50 target words per ear, starting with the right ear. The test is scored based on the number of correctly repeated words (50 points/ear). For assessing CAPD, a score $<35 \%$ for the right ear or $<35 \%$ for the left is considered abnormal. NU-6 List 5 was administered to the right ear and NU-6 List 6 was administered to the left ear.

\section{R-SPIN (Bilger et al, 1984)}

For the R-SPIN test, participants are told that they will hear a man say a sentence in an environment that sounds as if he is at a party. They are instructed to repeat back only the last word of the sentence that the man says and to guess if they are uncertain. All sentences are syntactically correct, and, in all cases, the final word is a monosyllabic noun; however, in half of the sentences, the final word is predictable from the context. The final word predictability is pseudorandomly varied from sentence to sentence. The test is administered diotically with both the target sentence and 12-talker babble delivered to the left and right ears at the same time. In this study, the target sentence was delivered at 50-dB SL (re: binaural SRT), beginning first with the babble set to be 47-dB SL (+3-dB SNR). Two lists of 50 sentences were administered, with List 1 being presented at $+3-\mathrm{dB}$ SNR and List 2 being presented at $0 \mathrm{~dB}$. This yielded four conditions: $+3-\mathrm{dB}$ high predictability, $+3-\mathrm{dB}$ low predictability, 0 - $\mathrm{dB}$ high predictability, and 0-dB low predictability. R-SPIN List 1 and 2 contain the same set of 50 target words, but the predictability of each target word is different across the two lists. For example, "spoon" occurs in a highpredictability context in List 1 ("Stir your coffee with a spoon") and in a low-predictability context in List 2 ("Bob could have known about the spoon"). According to the test developers, the two lists contain the same types of syllables, vowels, and consonants, and when administered at the same SNR, they are equivalent in terms of difficulty and reliability. Using the Corpus of Contemporary American English (https://corpus.byu.edu/coca/), we also confirmed that the lexical frequency of the high- and low-predictably target words was matched within a list.

The two SNR levels used in this study were selected based on pilot testing. Before starting the 0 - $\mathrm{dB}$ condition, the participant was first instructed that the task would be the same but that it might be more difficult to hear the man's voice. The percent correct for each condition was then calculated, with 25 being the highest possible raw score for each of the four conditions.

\section{Passage Comprehension (Woodcock-Johnson III Tests of Achievement)}

As a complement to the R-SPIN test, we administered an English reading comprehension that was taken from the Woodcock-Johnson III Tests of Achievement, in which a missing word must be filled in from context. Passage Comprehension evaluates written language comprehension at the sentence level by assessing the ability to make use of vocabulary, syntactic, and semantic knowledge to infer missing elements. For this study, we selected this test to evaluate the ability to make use of top-down linguistic knowledge in a nonauditory condition (Woodcock et al, 2001). For ages 14-30, the Passage Comprehension Test has high inter-test correlation $(r \geq 0.6)$ with the Oral Vocabulary, Oral Comprehension, Letter-Word Identification, and Spelling subtests of the Woodcock-Johnson III Tests of Achievement.

The Passage Comprehension Test was administered in a quiet room with the participant sitting across the table from the test administrator. During this test, the participant silently reads a sentence containing a missing word and then verbalizes the word that they think would best complete the sentence based on the context created by the other words in the sentence. This test is performed without a time limit. The test item was counted correct if it was included in the set of possible answers provided by the test manufacturers, or if it was a synonym of an answer provided by the manufacturer. The test includes a total of 38 items, beginning with simpler vocabulary and scaling to more advanced vocabulary. The first item administered was sentence 19 , which is considered to be at a Grade 10 level. When tabulating the final score, the participant received credit for the first 19 sentences. Standard scores and percentiles were then calculated according to the test manufacturer's guidelines.

\section{Statistical Analyses}

Percent scores were converted to rationalized arcsine transform units for statistical analysis (Studebaker, 1985). 
Rationalized arcsine transform unit linearizes percent scores, making the values better suited for analysis via linear tests (e.g., analyses of variance [ANOVAs], $t$-tests).

\section{RESULTS}

\section{Dichotic Digits, Competing Sentence, Time-Compressed Speech}

Participants performed at or near ceiling on the DDT and CST (Figure 1). For these tests, a mixed-model repeated measures ANOVA was performed using group (bilingual and monolingual) as the between-subjects factor, and ear (left versus right) as the within-subjects factor. All variables met the assumptions of sphericity.

For the DDT, there was the expected main effect of ear $\left[F_{(1,22)}=15.60, p=0.001\right.$, partial $\left.\eta^{2}=0.41\right]$, with lower accuracy for the left ear compared with the right ear. Two of the participants (one bilingual and one monolingual) fell below the 90\% cutoff for the left ear, but achieved perfect or near perfect scores in the right ear. For the DDT, neither the main effect of group $\left[F_{(1,22)}=0.05, p=0.83\right.$, partial $\left.\eta^{2}=0.002\right]$ nor the earby-group interaction was significant $\left[F_{(1,22)}=0.05\right.$, $p=0.82$, partial $\left.\eta^{2}=0.002\right]$.
For the CST, the main effect of ear was trending $\left[F_{(1,22)}=3.28, p=0.09\right.$, partial $\left.\eta^{2}=0.13\right]$, with performance being lower for the left ear relative to the right ear. In this case, three participants (all bilingual) scored below the $90 \%$ cutoff for the left ear. For this test, a group effect emerged $\left[F_{(1,22)}=5.30, p=0.03\right.$, partial $\left.\eta^{2}=0.19\right]$, but the interaction between ear and group was only trending $\left[F_{(1,22)}=2.27, p=0.12\right.$, partial $\left.\eta^{2}=0.11\right]$. Planned post hoc analysis revealed that the bilingual group underperformed the monolinguals on the left ear $\left[t_{(22)}=2.37, p=0.03, d=1.0\right]$, but that the groups were matched on the right ear condition, with both groups scoring $\sim 98 \%\left[t_{(22)}=0.53, p=0.59\right.$, $d=0.22$. For the bilingual group, the average score was $98.5 \%$ for the right ear $(\mathrm{SD}=1.67)$ compared with $93.92 \%$ for the left ear $(\mathrm{SD}=6.14)\left[t_{(11)}=2.02, p=0.07\right.$, $d=1.38]$.

For the Time-Compressed Speech With Reverberation Test, the effect of ear was trending $\left[F_{(1,22)}=\right.$ $3.65, p=0.07$, partial $\left.\eta^{2}=0.12\right]$; however, neither the main effect of group $\left[F_{(1,22)}=0.27, p=0.61\right.$, partial $\left.\eta^{2}=0.002\right]$ nor the ear-by-group interaction was significant $\left[F_{(1,22)}=0.04, p=0.84\right.$, partial $\left.\eta^{2}=0.002\right]$. On this test, all participants were in the clinically normal range.
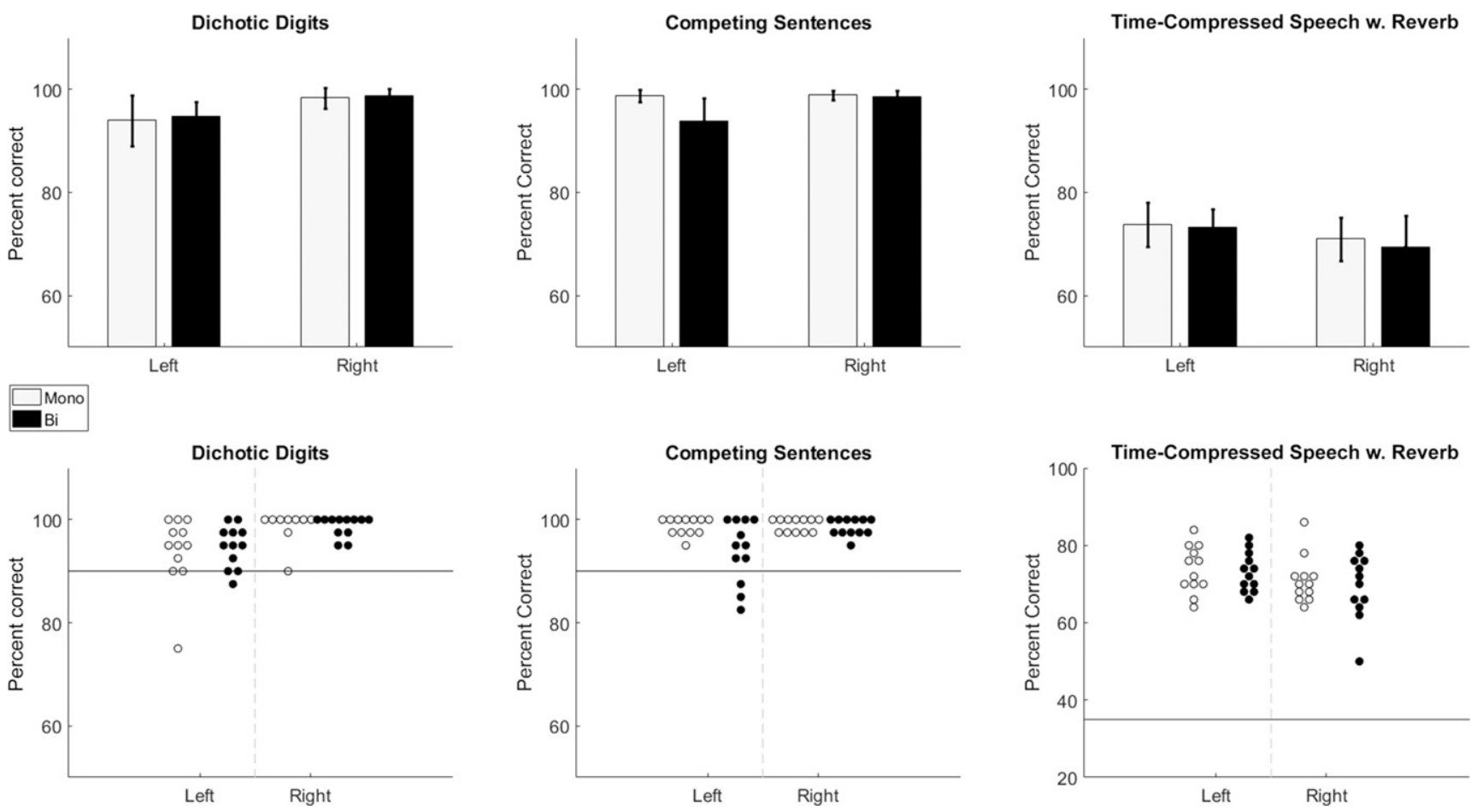

Figure 1. Comparisons between the monolingual (gray) and bilingual (black) groups on the Dichotic Digits, Competing Sentences, and Time-Compressed Speech with Reverberation Tests. In the top row, group means are plotted for each test, with error bars representing one standard error of the mean. In the bottom row, one-dimensional scatter plots show the distribution of scores across groups, ear, and tests. The horizontal line represents the cutoff score used for evaluating CAPD; scores below the line are considered abnormal. Note the number of perfect scores (100\%) for the right ear for both Dichotic Digits and Competing Sentences. In the case of Dichotic Digits, also note that a number of the data points are overlapping for the monolingual group for the right ear. 


\section{R-SPIN}

For the R-SPIN test, a mixed-model repeated measures ANOVA was performed using group (bilingual and monolingual) as the between-subjects factor and linguistic predictability (high versus low) as well as SNR (0 and $3 \mathrm{~dB})$ as within-subjects factors (Figure 2).

We start by reporting the within-subjects comparisons followed by the group comparisons: As expected, main effects of SNR and predictability were observed [SNR: $F_{(1,22)}=39.82, p<0.005$, partial $\eta^{2}=0.62$; predictability: $F_{(1,22)}=511.89, p<0.005$, partial $\eta^{2}=$ $0.96]$, with less accurate final word recognition observed in the 0-dB SNR condition compared with the 3-dB SNR condition (mean $[\mathrm{SD}]=70.5 \%[\mathrm{SD}=10.06]$ versus $79.17 \%$ [SD $=6.43]$ ) and also less accurate word recognition in the low-predictability compared with the highpredictability conditions $(58.92 \%$ [SD $=9.62]$ versus $90.75 \%$ [SD $=6.61]$ ). The facilitative influence of linguistic context, however, was different across the two SNR conditions [SNR $\times$ predictability interaction, $F_{(1,22)}=13.54, p=0.001$, partial $\left.\eta^{2}=0.38\right]$, with greater benefits of context observed for the $0-\mathrm{dB}$ SNR condition than the $3-\mathrm{dB}$ condition.

With respect to group comparisons, the overall main effect of group was trending toward significance $\left[F_{(1,22)}=\right.$ 3.05, $p=0.09$, partial $\left.\eta^{2}=0.12\right]$. Moreover, the SNR by group interaction was not significant $\left[F_{(1,22)}=\right.$ $0.21, p=0.65$, partial $\eta^{2}=0.009$ ], with both groups showing a performance decrement of $\sim 8 \%$ when the SNR dropped from 3 to $0 \mathrm{~dB}$. This can be seen visually in Figure 2; the distance between the square markers (3- $\mathrm{dB}$ condition) and the circle markers $(0 \mathrm{~dB})$ is matched for the two groups. Thus, on the R-SPIN test, our bilingual group was not inordinately affected by background noise compared with the monolinguals on the R-SPIN test. However, the bilingual group did differ from the monolingual group in terms of how much they benefitted from the linguistic predictability of the final word. In Figure 2, this manifests as difference in the slope of the lines connecting the low- and highpredictability conditions, with the slope being less steep for the bilingual group compared with the monolingual group. Collapsing across the two SNR conditions, the bilingual group had an average performance boost of $28.17 \%$ for the high-predictability sentences over the low-predictability sentences, compared with a $35.5 \%$ increase for the monolingual group. This is a small, yet, significant effect (predictability $\times$ group interaction $\left[F_{(1,22)}=14.27, p=0.001\right.$, partial $\left.\eta^{2}=0.39\right]$. Planned post hoc comparisons revealed that the groups had equivalent performance in the low-predictability condition $\left[t_{(22)}=-0.21, p=0.84, d=0.08\right]$ but differed in the high-predictability condition $\left[t_{(22)}=2.72, p=0.01\right.$,

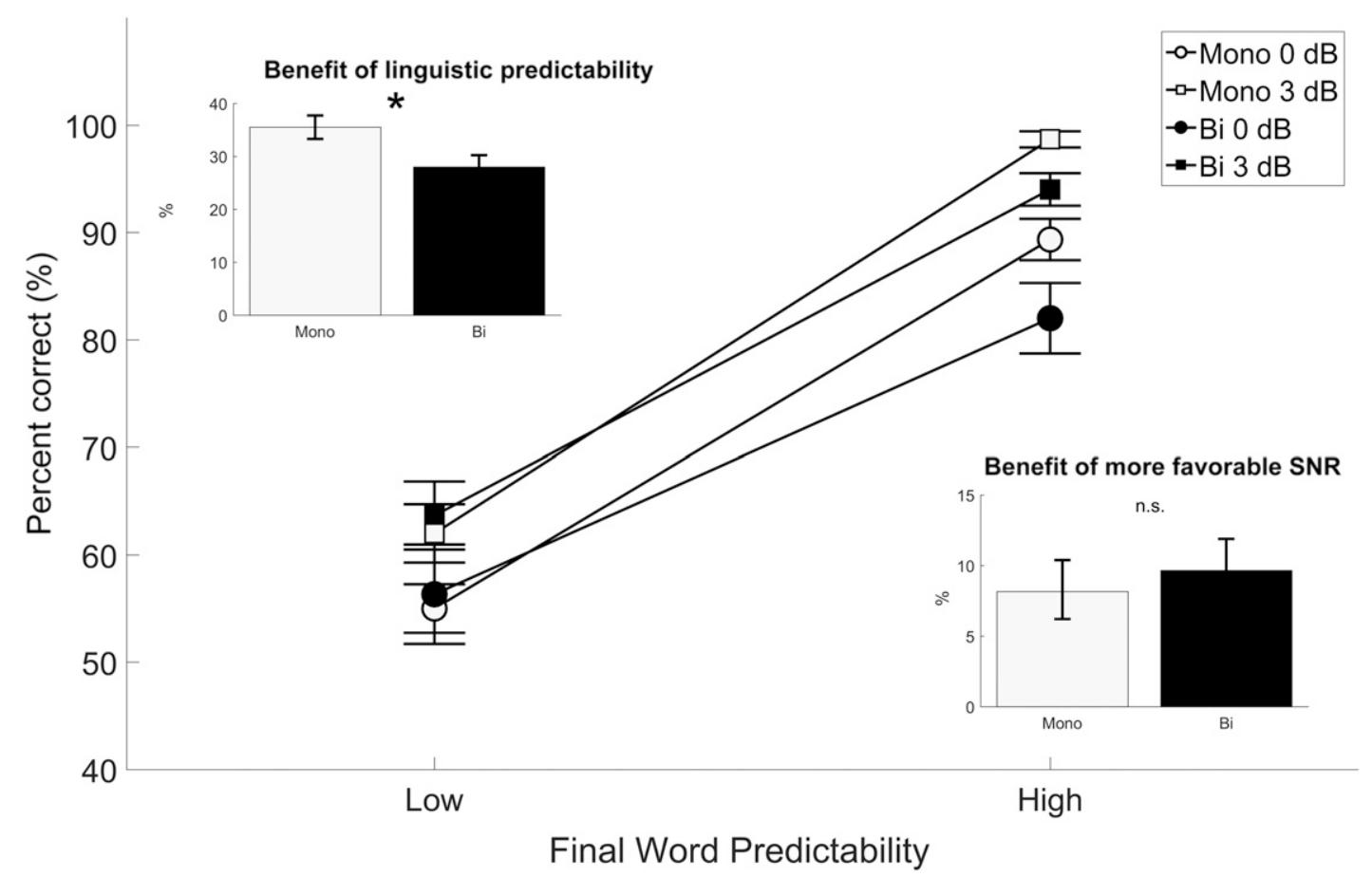

Figure 2. Comparisons between the monolingual (gray) and bilingual (black) groups on the R-SPIN test. Center plot: results of the four conditions for the two groups, with 0 -dB SNR conditions plotted with squares and the 3-dB condition plotted with circles. Across both the low- and high-predictability conditions (left and right, respectively), performance improved as the SNR increased from 0 to $3 \mathrm{~dB}$; however, both groups benefited to the same degree (bottom right inset panel). There was also a sharp improvement in performance when the final word could be deduced from context (high-predictability condition) compared with when it could not (low-predictability condition). In this case, the extent of the improvement was greater for the monolingual group than the bilingual group (top left inset panel). 
$d=1.2]$. Finally, the three-way interaction between group, SNR, and predictability was not significant $\left[F_{(1,1,1,22)}=0.01, p=0.91\right.$, partial $\left.\eta^{2}=0.001\right]$, suggesting that the differential effect of predictability for the two groups did not differ as a function of SNR.

\section{Passage Comprehension}

The two groups performed similarly on the Passage Comprehension Test $\left[t_{(22)}=0.10, p=0.92, d=\right.$ 0.04]. For the monolingual group, the average standard score was $113.50(\mathrm{SD}=7.78)$, with a range from 103 to 126 (58th to 96th percentile). For the bilingual group, the average standard score was 113.83 (SD = 8.34), with a range from 96 to 126 (39th to the 96th percentile).

\section{DISCUSSION}

$\mathrm{T}$ he ability to understand speech in noise is a complex process that reflects many different factors. Successful performance relies not only on the integrity of peripheral hearing and central auditory processes, but also on the ability to map the neural representation of the acoustic signal to a phonetic unit, match this phonetic information to lexical items, and use top-down linguistic knowledge including lexical, syntactic, semantic, and pragmatic information to interpret missing or obscured bottom-up information. Lagacé et al (2010) propose that the R-SPIN test has the advantage over other SIN tests by being able to dissociate whether the SUN weakness has its roots in auditory or language-based functions. Using the R-SPIN, together with a battery of three commonly used tests of CAPD, we do not find any evidence that bilingual listeners who self-rate as being proficient in the test language differ from monolingual listeners on their global auditory processing skills when using testing materials that involve speech stimuli. Instead, our constellation of findings suggest that differences between monolingual and bilingual individuals on the R-SPIN test reflect lessefficient top-down processing of speech. However, we are careful to point out that any apparent weakness or disadvantage observed in our bilingual group should not be construed as an impairment, given the overall highlevel performance seen across all tests.

In the following sections, we examine the degree to which the bilingual disadvantage in SUN reflects linguistic and not global auditory processes, the possibility that SUN weaknesses are an inevitable by-product of speaking two languages, how our findings fit within the debate on the advantages and disadvantages of speaking two languages, and the clinical implications of this line of work. Throughout these sections, we will highlight the novelty and limitations of our study and propose new avenues for investigating SUN in bilingual speakers, including the need for multimodal testing and other forms of SIN testing to further evaluate how bilingual listeners perform in acoustically degraded conditions.

\section{The Bilingual SUN Disadvantage Reflects Linguistic Not General Auditory Processing Abilities}

Our cohort of bilingual speakers was found to underperform in the R-SPIN test, but only under specific conditions. When manipulating the level of the background noise relative to the target sentence, we found that bilingual and monolingual listeners received a similar level of performance benefit when the SNR was more favorable, contradicting the claim that bilingual listeners are experiencing "auditory processing degradation" (Lucks Mendel and Widner, 2016). Instead, the outcomes of the R-SPIN test conditions suggest that bilingual listeners, even those who self-rate as being highly proficient in the test language, are weaker in using compensatory cues to aid their SUN, leading them to underperform on R-SPIN but only in conditions where the final (English) word can be restored from context. For some of the R-SPIN sentences, the contextual cues are so strong that the final word can be deduced without any clues about the auditory signal. However, when asked to fill in a missing word based on the context in a written sentence, with no time limits and no auditory clues, the two groups performed similarly, with both achieving high, near-ceiling scores on the Passage Comprehension WRMT-III Test. The bilingual group also performed on par with their monolingual counterparts in acoustically degraded conditions when the target English word was not predictable from context, as seen in the low-predictability conditions of the R-SPIN test and the Time-Compressed Speech with Reverberation test, in which listeners are prompted to repeat a target word without any aiding linguistic context ("Say the word __"). From this collective evidence, it appears that the bilingual college students in our sample were able to access and use top-down linguistic knowledge but that they may not have been able to capitalize on it to the same degree as monolingual listeners when sensory input was degraded (as in the R-SPIN test).

Consistent with the source of the bilingual disadvantage for SUN being top-down not bottom-up in nature, previous work has shown that the performance gap between bilingual and monolingual listeners does not increase as the amount of energetic masking increases (Rogers et al, 2006) or as the amount of time compression increases (Shi and Farooq, 2012), supporting the finding that our bilingual group is not inordinately affected by increasing levels of background noise on such tests. Moreover, when word- and sentence-level factors 
are stripped away from the SIN task, and the focus is shifted to identifying individual English phonemes, monolinguals and nonnative listeners have also been found to perform similarly (Cutler et al, 2004). In addition, there is evidence to suggest that noise tolerance levels, as measured by the Acceptable Noise Level test, are matched between bilingual and monolingual individuals, even when speech understanding is reduced (von Hapsburg and Bahng, 2006); however, other findings suggest that acceptable noise level scores are influenced by the listener's language background (Shi et al, 2015).

In our healthy young adult population, using the R-SPIN test, we isolated the bilingual disadvantage to top-down linguistic factors, although in our case, the disadvantage (relative to monolingual peers) is small. This weakness in leveraging top-down information is consistent with what has been found for listeners who are less proficient in the target language. For example, von Hapsburg and Bahng (2006) found that individuals who self-report as being moderately proficient in the test language (English) derived less benefit from context on the R-SPIN test than monolingual individuals, but those who self-report as having low proficiency in the target language show no benefit of linguistic predictability. Likewise, Mayo et al (1997) reported that bilingual listeners who acquire the target language late (after age 12), reach native-like proficiency on sentence recognition in quiet, but they do not derive the same benefit from cross-word context when the speech signals are presented in noise, again suggesting lessefficient top-down linguistic processing. A similar pattern of findings was reported for bilingual speakers who were asked to recall English passages in noise: compared with monolingual speakers, bilingual speakers with high self-rated English proficiency, but more wide-ranging ages of acquisition than the present study, did not derive the same benefit from the linguistic cues afforded by interconnected, linguistically related English sentences (Shi, 2012). These findings in diverse bilingual populations echo what has been shown in nonnative listeners who likewise have lessefficient use of top-down cues in their nonnative compared with their native language in background noise but also quiet conditions (Hervais-Adelman et al, 2014). Similar to the von Hapsburg and Bahng (2006) findings for bilingual speakers with (self-rated) low proficiency in the target language, Bradlow and Alexander (2007) reported that nonnative speakers could not take advantage of sentence-level context unless the speech signal was produced in clear speech, a type of speaking style often adopted by talkers in adverse communication environments in which the speaking rate is slowed and individual speech sounds are more discernable. Thus, whereas nonnative speakers may not benefit from top-down linguistic cues to facilitate word recogni- tion in noise until the speech signal becomes perceptually favorable, bilingual speakers with more native-like proficiency in the test language appear to be able to benefit from such cues, even in adverse listening conditions, but the benefit is not as great as a monolingual might achieve. Consistent with our findings, Schmidtke (2016) reported that bilingual listeners continued to underperform on R-SPIN sentences with high predictability relative to the monolingual listeners, even when using subsamples of bilingual and monolingual participants who were matched in language proficiency (as assessed by multiple standardized tests), suggesting that differences in language proficiency cannot fully account for these group differences. For a similar account, see Shi (2011).

Taken together, this combined evidence suggests that perceptual weaknesses for SUN that are observed in bilingual individuals are not necessarily due to a lack of knowledge in the target language, or a lack of linguistic knowledge more generally, but that they are instead more likely due to a linguistic system that underperforms when the bottom-up acoustic input is less reliable. However, a central limitation of our study is that we relied on self-report to estimate language proficiency. Moreover, although the high scores on the test of Passage Comprehension provide confirmatory evidence that the bilingual speakers in our sample have good mastery of English, this single test cannot provide a complete picture of language proficiency. Thus, although bilingual and monolingual groups were matched with respect to self-rated proficiency and performance on the Passage Comprehension Tests, it is premature to conclude that they are necessarily matched on all aspects of English language use and knowledge. Although it is a common practice to rely on self-report, Shi (2011) calls this practice into question, especially for late language learners who are more likely to overestimate their abilities. Another factor that is not adequately addressed by our study, or by the literature more generally, is how the quality of the exposure to the test language affects SIN performance and self-ratings of language proficiency, although the effect of language quantity and quality on language development are well recognized (Hart and Risley, 1995; Ramírez-Esparza et al, 2014). Our study findings, and comparisons to the broader literature, should, therefore, be interpreted with these limitations in mind.

\section{Are SUN Weaknesses Inevitable for Bilingual Listeners?}

It has recently been proposed that bilingual disadvantage for SUN is an inevitable consequence of being bilingual (Schmidtke, 2016). Under this theoretical framework, weaknesses on SUN tasks are not due to a lack of knowledge about the target language but they 
are instead considered to be by-product of knowing two languages and having to split one's time, as well as lexicon and phonetic inventory, across multiple languages. Thus, even when a bilingual speaker is a native speaker of the target language, she may still be at a communicative disadvantage, compared with monolinguals, when listening to SIN. Evidence supporting this "inevitability" viewpoint comes from current, well-accepted models of speech processing.

Current models of speech processing posit that upon hearing a (target) word, other similar sounding words and semantically related words are simultaneously activated in the mental lexicon (McClelland and Elman, 1986; Luce and Pisoni, 1998; Magnuson et al, 2007). These simultaneously activated words compete internally for recognition with the target word, and the listener must select the word that is deemed most plausible. When the bottom-up sensory signal is obscured by noise or otherwise degraded, the signal becomes less reliable, creating less certainty about what was said, and, this, it is theorized, leads to a greater number of candidate words being activated, which in turn increases the processing load. In such cases, the listener must rely more on nonauditory processes to consider the plausibility of each candidate word and discard those deemed least probable based on lexical knowledge, such as word frequency and other top-down linguistic information. A variety of evidence suggests that bilingual individuals face an increased processing load as a result of both languages being activated in parallel during speech processing (Weber and Cutler, 2004; Kerkhofs et al, 2006; Paulmann et al, 2006; Marian et al, 2008). This dual-activation results in both within- and across-language competitors being activated, producing a greater number of lexical competitors for bilingual compared with monolingual speakers. So, for example, when a monolingual English speaker hears the word "kite," phonological neighbors such as "bite" and "right" will be activated. However, for a bilingual listener, the set of activated (competing) words may also include non-English words with similar phonology. As an illustration, a German-English bilingual speaker may also activate words such as "kein" (none) or "weit" (far) (pronounced "kine" and "vite," respectively), when hearing "kite," creating more internal competition for bilingual listeners, who then must depend more on top-down knowledge to select the appropriate target word.

Further compounding these theorized lexical selection inefficiencies for bilingual individuals are lexical frequency effects. For all listeners, whether they are proficient in one or multiple languages, faster recognition and recall times are seen for frequently encountered words compared with less frequent words (Taft, 1979). However, in the case of bilingual individuals, the disadvantage for low-frequency words is exacerbated (Gollan et al, 2008). Consistent with this finding,
Schmidtke (2016) found that low-frequency words and words in less-predictable linguistic conditions were recognized less accurately by bilingual listeners compared with monolingual listeners on a modified version of R-SPIN. Gollan et al (2008) propose that this disadvantage for low-frequency words is the result of weaker connections between a lexical item and its phonological form, which arises because bilingual listeners activate each word in their lexicon less frequently than monolingual listeners simply because they know more words, on average, than monolingual individuals. For example, a bilingual individual and monolingual individual may encounter the English word "kite" the same number of times but because the bilingual individuals has a larger number of phonological representations within their lexicon by virtue of knowing two languages, the word "kite" will be processed/activated as if it is less frequent compared with the monolingual individual, leading to slower and less-efficient lexical recall for "kite." In further support of weaker lexical recall in bilinguals, bilingual speakers have also been shown to have lower verbal recall, slower reaction times on verbal recall measures, and poorer memory span for verbal information in both languages relative to monolinguals (Mägiste, 1979; Bialystok et al, 2009).

This literature, in combination with our findings, support the notion that bilingual individuals, even those who are native speakers of the test language, are at a comparative disadvantage, compared with monolinguals, when performing SUN tasks, as a consequence of lessefficient lexical retrieval. Our study illustrates that the disadvantage can, in some cases, be quite small.

\section{Bilingual Advantages and Disadvantages}

In interpreting our findings, we are mindful that our participant sample was limited in size, that we used self-ratings of language proficiency, and that although our dataset captured an array of different language families, it was by no means representative of the diversity of languages worldwide. Nevertheless, our small study provides an important data point in the larger discussion on the potential disadvantages of being bilingual by helping to delineate the conditions under which the bilingual disadvantage for SUN may or may not emerge for bilingual speakers who self-report as having native-like abilities in the test language. In addition, our study adds to the conversation on the potential benefits of being bilingual by providing evidence that the auditory processing advantages that have emerged for nonlinguistic stimuli (Bak et al, 2014; Krizman et al, 2016) and for auditory-evoked potentials to passively attended speech stimuli (Vihla et al, 2002; Krizman et al, 2012; 2015; Skoe et al, 2017) do not lead to any apparent behavioral benefits on the R-SPIN test nor on three tests routinely used to clinically assess 
CAPD. However, as seen in the present study, most of the participants (regardless of group) performed at or near ceiling on DDT and CST, suggesting that these linguistic-based tests of CAP lack the sensitivity to evaluate individual or group-level differences in CAP for high-performing (nonimpaired) listeners. In addition, although Lagacé et al (2011) theorize that the R-SPIN can be used to delineate auditory factors from linguistic factors, this claim has not undergone extensive scrutiny in the literature. Because it uses speech materials, we cannot rule out the possibility that the auditory processing component of the test (i.e., the manipulation of SNR in the R-SPIN) may reflect linguistic processing, at least to some degree. Phenomena such as the Ganong effect further illustrate the difficulty of separating linguistic and perceptual processes when the stimuli are speech or speech-like (Ganong, 1980). To better distill what specifically is being measured by the auditory dimension of the R-SPIN test, performance on the R-SPIN test should, in future investigations, be compared with performance nonlinguistic tests to determine whether an auditory processing advantage observed on nonlinguistic tests is associated with better performance for low-SNR R-SPIN conditions. Until then, we also leave open the possibility that the auditory processing advantages observed in previous work in bilinguals may counteract disadvantages for SUN by increasing the fidelity of the bottom-up signal, as suggested by Krizman et al (2016). Thus, enhanced basic auditory processing may help to level the playing field for processing SIN, although the data we present here do not provide evidence that either supports or refutes that possibility.

In addition, we did not observe evidence of a dichotic listening advantage in our bilingual participants. This stands in contrast to previous evidence of heightened dichotic processing in bilinguals (Soveri et al, 2011; Gresele et al, 2013). Gresele et al (2013), like the present study, used the DDT, but that study covered a much wider age range (18-59) and did not control for differences in educational level. These differences could account for the discrepant findings. Soveri et al (2011), in contrast, administered a phonemic version of a dichotic listening test in which two syllables (constantvowel) were played dichotically at the same intensity (dB level not reported) to Finnish-Swedish bilingual listeners and Swedish monolingual listeners between the ages of 30 and 74. In the "nonforced" condition, where the listener was not given explicit instructions as to which ear to attend to and was instead told to report back which syllable they heard best/first, both groups displayed a right-ear advantage and the groups did not differ in the degree of this advantage. This finding is consistent with what we observed for the DDT, in which the listener is asked to report back the numbers that they heard without selectively attending to one ear. In addition to the nonforced condition, Soveri et al (2011) included two other listening conditions, where the listeners were instructed to attend to either the right or the left ear and report back what they heard. When attending to the left ear in dichotic listening situations, the listener must inhibit this right-ear bias, and as a consequence, attending to the left ear is theorized to require more executive processing than attending to the right ear under dichotic stimulation (reviewed in Hugdahl et al, 2009). In the Soveri et al (2011) study, bilingual individuals had more accurate recall than the monolinguals for the left-attend and the right-attend conditions, which the authors took as evidence that bilingual individuals have stronger executive function.

As mentioned above, the DDT administered in our study does not include a selective attention condition (at least not as part of standardized procedures), which limits comparison with the Soveri et al (2011) study. However, we did administer the CST, which does provide a more comparable analog to the Soveri et al (2011) study. The CST uses full sentences not syllables, and unlike the dichotic syllables test used by Soveri et al (2011), the target sentence is presented at a lower intensity than the distractor. However, a right-ear advantage is still expected for the CST, even under conditions where the signal to the right ear is $15 \mathrm{~dB}$ less than the signal to the left ear (reviewed in Hugdahl et al, 2009). Using the CST, in our cohort of college students (who were younger than the Soveri et al, 2011 sample), we found that performance was at or near ceiling for both the left-attend and right-attend conditions, with the scores ranging from a low of $92.5 \%$ to a high of $100 \%$. Yet, even in the face of these high scores, the bilingual group still showed a small but statistically significant drop in performance compared with the monolingual group. This was most evident when the task was to focus on the left ear compared with the right ear, consistent with the right-ear advantage for this task. The monolingual group, by contrast, showed no ear bias by performing at or near ceiling on both conditions. One interpretation of this finding is that the monolingual group in our dataset has more refined executive function than the bilingual group. Thus, whereas previous studies suggest that bilingual individuals may be able to draw on more refined executive skills to outperform monolingual individuals on selective auditory attention tasks that involve nonlinguistic stimuli (Bak et al, 2014) or simple linguistic stimuli such as numbers (Krizman et al, 2012) and syllables (Soveri et al, 2011), our findings suggest that the purported bilingual advantage in harnessing executive function does not necessarily transfer to more linguistically challenging stimuli or that it may not manifest at all. A different, yet not contradictory, interpretation is that listening tasks that require more cognitive control expose an 
underlying (subtle, in our case) difference in linguistic processing.

Although our small study, like recent more largescale studies of bilinguals (Paap et al, 2014; 2015), does not provide direct or even indirect evidence of enhanced executive function in our young adult bilingual cohort, this does not necessarily discount the possibility that bilingualism may advantage certain aspects of executive function and/or auditory processing at points in life (Bialystok et al, 2005). Future studies that include comprehensive assessments of language, executive, and auditory function are needed to more fully explore the linguistic and nonlinguistic conditions under which selective listening advantages emerge for bilingual speakers at different points in life.

\section{Is the Bilingual Disadvantage in Noise Modality Specific?}

We now turn to the question of whether the bilingual disadvantage is modality specific. In the case of dyslexia, difficulties understanding SIN has been theorized to be the outcome of a sensory-wide difficulty with inhibiting noise for both auditory (speech and nonspeech), as well as visual conditions (Sperling et al, 2005). For musically trained populations, advantages have been seen for adverse (i.e., degraded or distorted) conditions across both visual and auditory modalities (Anaya et al, 2016). In the case of bilingual individuals, current data suggest that the disadvantages that bilingual speakers face in noise are specific to speech and that they do not generalize to nonspeech signals (Krizman et al, 2016); however, more work needs to be done to examine whether the bilingual weakness in noise is specific to language within the auditory modality or whether it might be more sensory pervasive and extend to written forms of language.

In the current investigation, we incorporated a visual test of language processing (Passage Comprehension) that assesses the ability to use top-down linguistic information. Although both the auditory-based R-SPIN test and visual-based Passage Comprehension assess top-down language skills, the two tests are not true analogs (Bellis et al, 2011). In the case of R-SPIN, the sensory input was degraded, but for the Passage Comprehension Test, it (i.e., visual input) was not. Although we did not administer the R-SPIN test in a "quiet" condition without multitalker babble, we can infer from the results of the Time-Compressed Speech Test with Reverberation and previous work in bilingual and trilingual speakers (Mayo et al, 1997; Rogers et al, 2006; Tabri et al, 2011; Shi, 2012) that the groups would likely perform similarly in an R-SPIN condition in quiet, at least in the low-predictability condition. Another way in which the R-SPIN and Passage Comprehension Tests differ is in their performance loads.
The Passage Comprehension Test is administered without a time limit. Although the R-SPIN test is not a timed test, per se, the listener is expected to keep to the pace that the test materials are delivered in the digital recordings. The comparatively slower pace of the Passage Comprehension Test may have allowed the bilingual individuals to reach monolingual-like levels. Future research should consider incorporating a visual analog of the R-SPIN test in which the text is difficult to make out (e.g., blurred, faintly colored text, and visual masker) to illuminate whether this bilingual weakness with top-down information under degraded condition is specific to auditory input (Zekveld and Kramer, 2014; Anaya et al, 2016). An investigation using noise in both auditory and visual would not only shed light on mechanisms of why bilingual speakers are likely to underperform on SUN tasks but also help to guide clinical recommendations for accommodating such weakness.

\section{Clinical Implications}

Specialists in all areas of health care, including audiologists and speech-language pathologists, are now treating a larger percentage of bilingual patients. Census reports from 2010 estimated that roughly $20 \%$ of the US population is bilingual, with a nearly $40 \%$ rise in bilingualism between 1980 and 2010; however, clinical services, and the number of bilingual audiologists, have not necessarily kept pace with the growing bilingual population. The challenge with caring for bilingual populations is that most clinical norming criteria are based on monolingual datasets and use English-only materials, and therefore do not take into account that bilingual individuals might have different performance baselines (von Hapsburg and Peña, 2002). Our findings emphasize that (a) bilinguals listeners, even those with normal hearing, no noticeable accent, and who consider themselves to have high proficiency in English, may underperform on English SUN tests, under certain conditions and (b) the choice of test materials is critical. Shi (2011) recommended that for English-dominant, early bilinguals that proficiency ratings of 8 or better (out of 10) are required for using monolingual normative values for the NU-6 test, although our findings suggest that this recommendation does not generalize to all SIN and CAP tests. The idea of developing bilingualspecific norms, and language-specific materials, for audiological use, as well as training more bilingual audiologists, is intuitively appealing; however, it is an inherently complex, potentially fraught process, given the diversity of bilingual backgrounds (different languages, different proficiency levels, etc.) that may be encountered in a clinical setting. Another area that needs further exploration is the potential impact of having a bilingual audiologist administer and score SIN tests. In the case of the present study, the test 
administrator was bilingual, and given that test scoring for many audiological tests is contingent on the test administrator's perception of what the patient says, this could be viewed as a potential confound.

An alternative to developing language-specific norms is to use a test such as the R-SPIN that can (theoretically) dissociate auditory and linguistic factors and/or to use SIN tests that have a low linguistic load. A recent study suggests that children who learn two languages simultaneously can achieve the same level of performance on SIN tests as SES-matched children, when the age of English acquisition is matched and speech materials use simple vocabulary that minimizes linguistic load/bias (Reetzke et al, 2016). Reetzke et al (2016) replicated this finding across multiple SNR conditions, under different types of maskers, and in auditory-only and audiovisual conditions, providing strong converging evidence that bilingual and monolingual individuals can achieve similar levels of performance in noise when linguistic materials are adequately controlled. For assessing central auditory function in bilingual (as well monolingual) speakers, there is also value in incorporating nonlinguistic measures of central auditory function (Moore et al, 2010; Ludwig et al, 2014), using SIN tests that do not rely on a direct report of speech understanding, such as electrophysiological testing (Krizman et al, 2012), creating tests that allow for a greater spread of performance among nonimpaired listeners, and/or administering subjective tests of noise tolerance and/or listening effort in noise (von Hapsburg and Bahng, 2006; Shi et al, 2015).

However, a first step in developing more bilingualfocused care is to establish more widespread clinical recognition and scientific exploration of the specific advantages and disadvantages that bilinguals may display on tests that are routinely administered in the evaluation of central and peripheral auditory function. This will be key for developing more tailored strategies for counseling and remediation in disordered bilingual populations, as well as developing hearing conservation programs that address the specific difficulties faced by bilingual individuals.

Acknowledgments. This study was completed as partial fulfillment of the second author's Au.D. Degree. We extend our thanks to Parker Tichko for his comments on an earlier version of this manuscript and Kelly Linehan for her assistance with data coding and copyediting.

\section{REFERENCES}

Anaya EM, Pisoni DB, Kronenberger WG. (2016) Long-term musical experience and auditory and visual perceptual abilities under adverse conditions. J Acoust Soc Am 140(3):2074-2081.
Bak TH, Vega-Mendoza M, Sorace A. (2014) Never too late? An advantage on tests of auditory attention extends to late bilinguals. Front Psychol 5:485.

Bellis TJ, Billiet C, Ross J. (2011) The utility of visual analogs of central auditory tests in the differential diagnosis of (central) auditory processing disorder and attention deficit hyperactivity disorder. J Am Acad Audiol 22(8):501-514.

Bialystok E. (2015) Bilingualism and the development of executive function: the role of attention. Child Dev Perspect 9(2):117-121.

Bialystok E, Craik FI, Green DW, Gollan TH. (2009) Bilingual minds. Psychol Sci Public Interest 10(3):89-129.

Bialystok E, Martin MM, Viswanathan M. (2005) Bilingualism across the lifespan: the rise and fall of inhibitory control. Int $J$ Biling 9(1):103-119.

Bilger RC, Nuetzel JM, Rabinowitz WM, Rzeczkowski C. (1984) Standardization of a test of speech perception in noise. $J$ Speech Hear Res 27(1):32-48.

Bradlow AR, Alexander JA. (2007) Semantic and phonetic enhancements for speech-in-noise recognition by native and nonnative listeners. J Acoust Soc Am 121(4):2339-2349.

Costa A, Hernández M, Costa-Faidella J, Sebastián-Gallés N. (2009) On the bilingual advantage in conflict processing: now you see it, now you don't. Cognition 113(2):135-149.

Cutler A, Weber A, Smits R, Cooper N. (2004) Patterns of English phoneme confusions by native and non-native listeners. $J$ Acoust Soc Am 116(6):3668-3678.

Ganong WF 3rd. (1980) Phonetic categorization in auditory word perception. J Exp Psychol Hum Percept Perform 6(1):110-125.

García-Sierra A, Ramírez-Esparza N, Silva-Pereyra J, Siard J, Champlin CA. (2012) Assessing the double phonemic representation in bilingual speakers of Spanish and English: an electrophysiological study. Brain Lang 121(3):194-205.

Gollan TH, Montoya RI, Cera C, Sandoval TC. (2008) More use almost always a means a smaller frequency effect: aging, bilingualism, and the weaker links hypothesis. J Mem Lang 58(3): 787-814.

Green DW. (1998) Mental control of the bilingual lexico-semantic system. Biling Lang Cogn 1(2):67-81.

Gresele AD, Garcia MV, Torres EM, Santos SN, Costa MJ. (2013) Bilingualism and auditory processing abilities: performance of adults in dichotic listening tests. CoDAS 25(6):506-512.

Hart B, Risley TR. (1995) Meaningful Differences in the Everyday Experience of Young American Children. Baltimore, MD: Paul H. Brookes Publishing.

Hervais-Adelman A, Pefkou M, Golestani N. (2014) Bilingual speech-in-noise: neural bases of semantic context use in the native language. Brain Lang 132:1-6.

Hugdahl K, Westerhausen R, Alho K, Medvedev S, Laine M, Hämäläinen H. (2009) Attention and cognitive control: unfolding the dichotic listening story. Scand J Psychol 50(1):11-22.

Kerkhofs R, Dijkstra T, Chwilla DJ, de Bruijn ER. (2006) Testing a model for bilingual semantic priming with interlingual homographs: RT and N400 effects. Brain Res 1068(1):170-183.

Killion MC, Niquette PA, Gudmundsen GI, Revit LJ, Banerjee S. (2004) Development of a quick speech-in-noise test for measuring 
signal-to-noise ratio loss in normal-hearing and hearing-impaired listeners. J Acoust Soc Am 116(4 Pt 1):2395-2405. Erratum in: $J$ Acoust Soc Am 2006;119(3):1888.

Krizman J, Bradlow AR, Lam SS-Y, Kraus N. (2016) How bilinguals listen in noise: linguistic and non-linguistic factors. Biling Lang Cogn 20:842-843.

Krizman J, Marian V, Shook A, Skoe E, Kraus N. (2012) Subcortical encoding of sound is enhanced in bilinguals and relates to executive function advantages. Proc Natl Acad Sci USA 109(20): 7877-7881.

Krizman J, Skoe E, Marian V, Kraus N. (2014) Bilingualism increases neural response consistency and attentional control: evidence for sensory and cognitive coupling. Brain Lang 128(1): $34-40$.

Krizman J, Slater J, Skoe E, Marian V, Kraus N. (2015) Neural processing of speech in children is influenced by extent of bilingual experience. Neurosci Lett 585:48-53.

Lagacé J, Jutras B, Gagné J-P. (2010) Auditory processing disorder and speech perception problems in noise: finding the underlying origin. Am J Audiol 19(1):17-25.

Lagacé J, Jutras B, Giguère C, Gagné J-P. (2011) Speech perception in noise: exploring the effect of linguistic context in children with and without auditory processing disorder. Int $J$ Audiol 50(6): 385-395.

Luce PA, Pisoni DB. (1998) Recognizing spoken words: the neighborhood activation model. Ear Hear 19(1):1-36.

Lucks Mendel L, Widner H. (2016) Speech perception in noise for bilingual listeners with normal hearing. Int $J$ Audiol 55(2): 126-134

Ludwig AA, Fuchs M, Kruse E, Uhlig B, Kotz SA, Rübsamen R. (2014) Auditory processing disorders with and without central auditory discrimination deficits. J Assoc Res Otolaryngol 15(3): 441-464.

Mägiste E. (1979) The competing language systems of the multilingual: A developmental study of decoding and encoding processes. J Verbal Learn Verbal Behav 18(1):79-89.

Magnuson JS, Dixon JA, Tanenhaus MK, Aslin RN. (2007) The dynamics of lexical competition during spoken word recognition. Cogn Sci 31(1):133-156.

Marian V, Blumenfeld HK, Boukrina OV. (2008) Sensitivity to phonological similarity within and across languages. J Psycholinguist Res 37(3):141-170.

Mayo LH, Florentine M, Buus S. (1997) Age of second-language acquisition and perception of speech in noise. $J$ Speech Lang Hear Res 40(3):686-693.

McClelland JL, Elman JL. (1986) The TRACE model of speech perception. Cognit Psychol 18(1):1-86.

Montagni I, Peru A. (2011) Effects of language and music learning on pitch and duration perception: an experimental investigation. Int J Psychol Behav Sci 1(1):33-40.

Moore DR, Ferguson MA, Edmondson-Jones AM, Ratib S, Riley A. (2010) Nature of auditory processing disorder in children. Pediatrics 126(2):e382-e390.

Musiek FE. (1983) Assessment of central auditory dysfunction: the dichotic digit test revisited. Ear Hear 4(2):79-83.
Nilsson M, Soli SD, Sullivan JA. (1994) Development of the hearing in noise test for the measurement of speech reception thresholds in quiet and in noise. J Acoust Soc Am 95(2):1085-1099.

Paap KR, Johnson HA, Sawi O. (2014) Are bilingual advantages dependent upon specific tasks or specific bilingual experiences? $J$ Cogn Psychol 26(6):615-639.

Paap KR, Johnson HA, Sawi O. (2015) Bilingual advantages in executive functioning either do not exist or are restricted to very specific and undetermined circumstances. Cortex 69:265-278.

Parbery-Clark A, Skoe E, Lam C, Kraus N. (2009) Musician enhancement for speech-in-noise. Ear Hear 30(6):653-661.

Paulmann S, Elston-Güttler KE, Gunter TC, Kotz SA. (2006) Is bilingual lexical access influenced by language context? Neuroreport 17(7):727-731

Ramírez-Esparza N, García-Sierra A, Kuhl PK. (2014) Look who's talking: speech style and social context in language input to infants are linked to concurrent and future speech development. Dev Sci 17(6):880-891.

Reetzke R, Lam BP-W, Xie Z, Sheng L, Chandrasekaran B. (2016) Effect of simultaneous bilingualism on speech intelligibility across different masker types, modalities, and signal-to-noise ratios in school-age children. PLoS One 11(12):e0168048.

Rogers CL, Lister JJ, Febo DM, Besing JM, Abrams HB. (2006) Effects of bilingualism, noise, and reverberation on speech perception by listeners with normal hearing. Appl Psychol 27(3):465-485.

Schmidtke J. (2016) The bilingual disadvantage in speech understanding in noise is likely a frequency effect related to reduced language exposure. Front Psychol 7:678.

Shi L-F. (2010) Perception of acoustically degraded sentences in bilingual listeners who differ in age of English acquisition. $J$ Speech Lang Hear Res 53(4):821-835.

Shi LF. (2011) How "proficient" is proficient? Subjective proficiency as a predictor of bilingual listeners' recognition of English words. Am J Audiol 20(1):19-32.

Shi LF. (2012) Contribution of linguistic variables to bilingual listeners' perception of degraded English sentences. J Speech Lang Hear Res 55(1):219-234.

Shi L-F, Azcona G, Buten L. (2015) Acceptance noise level: effects of the speech signal, babble, and listener language. $J$ Speech Lang Hear Res 58(2):497-508.

Shi L-F, Farooq N. (2012) Bilingual listeners' perception of temporally manipulated English passages. J Speech Lang Hear Res 55(1):125-138.

Skoe E, Burakiewicz E, Figueiredo M, Hardin M. (2017) Basic neural processing of sound in adults is influenced by bilingual experience. Neuroscience 349:278-290.

Slater J, Kraus N. (2016) The role of rhythm in perceiving speech in noise: a comparison of percussionists, vocalists and non-musicians. Cogn Process 17(1):79-87.

Slater J, Skoe E, Strait DL, O'Connell S, Thompson E, Kraus N. (2015) Music training improves speech-in-noise perception: longitudinal evidence from a community-based music program. Behav Brain Res 291:244-252.

Soveri A, Laine M, Hämäläinen H, Hugdahl K. (2011) Bilingual advantage in attentional control: evidence from the forced-attention dichotic listening paradigm. Biling Lang Cogn 14(3):371-378. 
Sperling AJ, Lu ZL, Manis FR, Seidenberg MS. (2005) Deficits in perceptual noise exclusion in developmental dyslexia. Nat Neurosci 8(7):862-863.

Studebaker GA. (1985) A "rationalized" arcsine transform. $J$ Speech Hear Res 28(3):455-462.

Tabri D, Abou Chacra KM, Pring T. (2011) Speech perception in noise by monolingual, bilingual and trilingual listeners. Int $J$ Lang Commun Disord 46(4):411-422.

Taft M. (1979) Recognition of affixed words and the word frequency effect. Mem Cognit 7(4):263-272.

Vihla M, Kiviniemi K, Salmelin R. (2002) Auditory cortical activation in Finnish and Swedish speaking Finns: a magnetoencephalographic study. Neurosci Lett 322(3):141-144.

von Hapsburg D, Bahng J. (2006) Acceptance of background noise levels in bilingual (Korean-English) listeners. J Am Acad Audiol 17(9):649-658.

von Hapsburg D, Peña ED. (2002) Understanding bilingualism and its impact on speech audiometry. J Speech Lang Hear Res 45(1):202-213.
Weber A, Cutler A. (2004) Lexical competition in non-native spokenword recognition. J Mem Lang 50(1):1-25.

Willeford JA. (1978) Sentence tests of central auditory dysfunction. In: Katz J, Medwetsky L, Burkard R, eds. Handbook of Clinical Audiology. Philadelphia, PA: Wolters Kluwer Health.

Wilson RH, Abrams HB, Pillion AL. (2003) A word-recognition task in multitalker babble using a descending presentation mode from $24 \mathrm{~dB}$ to $0 \mathrm{~dB}$ signal to babble. J Rehabil Res Dev 40(4): $321-327$.

Wilson RH, Preece JP, Salamon DL, Sperry JL, Bornstein SP. (1994) Effects of time compression and time compression plus reverberation on the intelligibility of Northwestern University Auditory Test No. 6. J Am Acad Audiol 5(4):269-277.

Woodcock R, Mather N, McGrew K. (2001) Woodcock-Johnson III Tests of Cognitive Abilities Examiner's Manual. Itasca, IL: Riverside.

Zekveld AA, Kramer SE. (2014) Cognitive processing load across a wide range of listening conditions: insights from pupillometry. Psychophysiology 51(3):277-284. 